OPEN ACCESS

Edited by:

Jennifer Elizabeth Cowan,

National Institutes of Health $(\mathrm{NIH})$,

United States

Reviewed by:

Laijun Lai,

University of Connecticut,

United States

Kristin Hogquist,

University of Minnesota Twin Cities,

United States

*Correspondence:

Magali Irla

Magali.Irla@inserm.fr

Specialty section:

This article was submitted to

T Cell Biology,

a section of the journal

Frontiers in Immunology

Received: 29 October 2020

Accepted: 07 December 2020

Published: 22 January 2021

Citation:

Irla M (2021) RANK Signaling in the Differentiation and Regeneration of

Thymic Epithelial Cells.

Front. Immunol. 11:623265. doi: 10.3389/fimmu.2020.623265

\section{RANK Signaling in the Differentiation and Regeneration of Thymic Epithelial Cells}

\author{
Magali Irla* \\ Aix Marseille Univ, CNRS, INSERM, CIML, Centre d'Immunologie de Marseille-Luminy, Marseille, France
}

Thymic epithelial cells (TECs) provide essential clues for the proliferation, survival, migration, and differentiation of thymocytes. Recent advances in mouse and human have revealed that TECs constitute a highly heterogeneous cell population with distinct functional properties. Importantly, TECs are sensitive to thymic damages engendered by myeloablative conditioning regimen used for bone marrow transplantation. These detrimental effects on TECs delay de novo T-cell production, which can increase the risk of morbidity and mortality in many patients. Alike that TECs guide the development of thymocytes, reciprocally thymocytes control the differentiation and organization of TECs. These bidirectional interactions are referred to as thymic crosstalk. The tumor necrosis factor receptor superfamily (TNFRSF) member, receptor activator of nuclear factor kappa$\mathrm{B}$ (RANK) and its cognate ligand RANKL have emerged as key players of the crosstalk between TECs and thymocytes. RANKL, mainly provided by positively selected CD4 ${ }^{+}$ thymocytes and a subset of group 3 innate lymphoid cells, controls mTEC proliferation/ differentiation and TEC regeneration. In this review, I discuss recent advances that have unraveled the high heterogeneity of TECs and the implication of the RANK-RANKL signaling axis in TEC differentiation and regeneration. Targeting this cell-signaling pathway opens novel therapeutic perspectives to recover TEC function and T-cell production.

Keywords: bone marrow transplantation, central tolerance, receptor activator of nuclear factor kappa-B, thymic crosstalk, thymic epithelial cells, thymic regeneration

\section{INTRODUCTION}

The thymus supports the generation of distinct T-cell subsets such as conventional $\mathrm{CD} 4^{+}$and $\mathrm{CD} 8^{+} \mathrm{T}$ cells, Foxp $3^{+}$regulatory T cells, $\gamma \delta \mathrm{T}$ cells, and invariant natural killer T cells (iNKT). The development of these different T-cell subsets depends on stromal niches composed of thymic epithelial cells (TECs). TECs control T-cell development from the entry of T-cell progenitors to the egress of mature $\mathrm{T}$ cells. According to their anatomical localization and functional properties, TECs are subdivided into two main populations: cortical TECs (cTECs) and medullary TECs (mTECs). cTECs support the initial stages of T-cell development, including T-cell progenitor homing, T-cell lineage commitment, the expansion of immature thymocytes, death by neglect of thymocytes that do not recognize peptide-MHC complexes and positive selection of thymocytes into $\mathrm{CD} 4^{+}$and $\mathrm{CD} 8^{+} \mathrm{T}$ cells. By contrast, mTECs control 
late stages of T-cell development, mainly the induction of selftolerance characterized by the clonal deletion of autoreactive thymocytes and $\mathrm{CD}^{+}$thymocyte diversion into the Foxp $3^{+}$ regulatory $\mathrm{T}$-cell lineage. Conversely, thymocytes control TEC expansion and differentiation. These bidirectional interactions between thymocytes and TECs are termed thymic crosstalk (1-3).

\section{TEC HETEROGENEITY IN MOUSE AND HUMAN}

Historically, cTECs and mTECs were identified by histology using distinct markers such as cytokeratin 8 for cTECs and cytokeratin-5 and -14 for mTECs (4). TEC identification by flow cytometry on enzymatically-disaggregated thymus has greatly aided in studying TEC heterogeneity and functionality. TECs are non-hematopoietic cells, which express the Epithelial Cell Adhesion Molecule (EpCAM), and are generally identified as $\mathrm{CD} 45^{-} \mathrm{EpCAM}^{+}$. TECs can be further segregated into cTECs and mTECs based on the detection of Ly51 and reactivity to the lectin Ulex Europaeus Agglutinin 1 (UEA-1), respectively. cTECs and mTECs have distinct phenotypic and functional properties. Recent advances based on single-cell transcriptomic analyses have highlighted that TECs constitute a more diverse and dynamic population than previously thought.

\section{FEATURES OF CORTICAL TECs}

cTECs express several molecules that govern the initial stages of T-cell development. They express CXCL12 and CCL25 chemokines that guide the homing of T-cell progenitors into the thymus $(5,6)$. cTECs also express the NOTCH ligand Delta-like 4 (DLL4), which induces the engagement of progenitors into the T-cell lineage $(7,8)$. Moreover, they express IL-7 and stem cell factor (SCF) cytokines that promote the survival and proliferation of immature thymocytes (9). They are equipped with protein degradation machineries important for the positive selection of $\mathrm{CD} 4^{+}$thymocytes such as the lysosomal endopeptidase cathepsin L (encoded by Ctsl) and the thymus-specific serine protease TSSP (encoded by Prss16) that contributes to MHC class II-associated self-peptide generation (10). They also express the thymoproteasome subunit $\beta 5 \mathrm{t}$ (encoded by Psmb11), which produces MHC class I-associated self-peptides required for the positive selection of $\mathrm{CD}^{+}$thymocytes (11).

cTECs are heterogeneous based on the expression level of MHCII, CD40, DLL4, and IL-7. Intriguingly, a cTEC subset specific of the perinatal thymus termed perinatal cTECs has been identified by single-cell transcriptomics (12). These cells, representing one-third of all TECs at 1 week of age, are highly proliferative and express synaptogyrin 1 (Syngr1) and G proteincoupled estrogen receptor 1 (Gper1) in addition to classical cTEC markers. Furthermore, by enveloping many viable double-positive (DP) thymocytes, a fraction of cTECs can form multi-cellular complexes called thymic nurse cells (TNCs) (13). TNCs likely provide a microenvironment favorable to secondary TCR $\alpha$ rearrangements in long-lived DP thymocytes, thereby optimizing TCR repertoire selection (14). Although TNCs remain poorly characterized, they exhibit a distinct gene expression profile characterized by high expression of CXCL12 and TSSP. TNCs thus constitute a cTEC subpopulation with distinct morphological and functional properties. Given that cTECs ensure multiple functions such as i) lymphoid progenitor homing, ii) T-cell lineage commitment, iii) immature thymocyte expansion, and iv) positive selection of thymocytes, it is likely that cTECs contain discrete functional subsets. Further investigations are required to clarify cTEC heterogeneity. Their development is regulated by signals provided by developing thymocytes. Human CD3€ transgenic mice (tg€26 mice), in which T-cell development is blocked at the early DN1 stage, have a disorganized cortex with cTECs arrested at the $\mathrm{CD}^{-} \mathrm{MHCII}^{\mathrm{lo}}$ stage $(15,16)$. However, the transplantation of tge26 recipients with bone marrow cells from $\mathrm{Rag}^{-/-}$mice, exhibiting a subsequent block at the DN3 stage, restores the cortical organization $(4,17)$. Furthermore, cTECs with a $\mathrm{CD} 40^{+} \mathrm{MHCII}^{\text {hi }}$ phenotype develop in the thymus of $\mathrm{Ragl}^{-/-}$mice (16). Thus, cTEC development requires signals from thymocytes beyond the DN1 stage. Nevertheless, the cell-signaling pathways responsible for their development remain to be determined.

\section{FEATURES OF MEDULLARY TECs}

Compared to cTECs, mTECs are better characterized, likely because they are more abundant. mTECs have the unique ability to express up to $85 \%-90 \%$ of the genome and virtually all protein-coding genes (18). This promiscuous gene expression program is induced by the autoimmune regulator (Aire) and the transcription factor Fez family zinc finger 2 (Fezf2) $(18,19)$. mTECs contain two main subsets identified on MHCII and $\mathrm{CD} 80$ cell surface expression levels: $\mathrm{MHCII}^{\mathrm{lo}} \mathrm{CD}^{\circ} 0^{\mathrm{lo}}\left(\mathrm{mTEC}^{\mathrm{lo}}\right)$ and MHCII ${ }^{\text {hi }} \mathrm{CD} 80^{\text {hi }}$ (mTEC ${ }^{\text {hi }}$ ) (20). These two subsets are heterogeneous based on distinct markers and functional properties. mTEC $^{\text {lo }}$ contain mTEC $^{\text {hi }}$ precursors expressing alpha-6 integrin (Itga6) and Sca1 (Ly6a) (21-23). They also comprise CCL2 $1^{+}$mTECs implicated in the migration of positively-selected thymocytes into the medulla (24). Cell fate mapping studies have identified that mTEC $^{\text {lo }}$ contain post-Aire cells characterized by the loss of Aire protein and low surface levels of MHCII and CD80 molecules (25-27). Another subset of terminally differentiated mTECs closely resembling the gut chemosensory epithelial tuft cells are also present in mTEC $^{\text {lo }}$ $(28,29)$. These cells express the doublecortin-like kinase 1 (Dclk1) marker and the transcription factor Pou2f3. Thus, the mTEC $^{\text {lo }}$ compartment is particularly heterogeneous, containing not only mTEC $^{\text {hi }}$ precursors but also CCL $21^{+}$, post-Aire and tuft-like mTECs. The mTEC $^{\text {hi }}$ compartment is also diverse, containing Aire ${ }^{-} \mathrm{Fezf} 2^{+}$and Aire ${ }^{+} \mathrm{Fezf} 2^{+}$subsets.

Single-cell transcriptomic analyses have identified dozens of TEC subsets, including perinatal cTECs, mature cTECs, mTEC progenitors, Aire ${ }^{+}$, post-Aire and tuft-like mTECs $(12,28,30)$. Among them, two other minor subsets termed neuronal and structural TECs have been identified based on their expression signatures associated with neurotransmitters and extracellular matrix such as collagens and proteoglycans (12). Further investigations are required to define their anatomical localization 
and function. Interestingly, a subset of proliferating mTECs expresses substantial levels of Aire, suggesting that it corresponds to a maturational stage just before Aire ${ }^{+}$mature mTECs $(12,30)$.

In humans, cTECs and mTECs are defined as EPCAM ${ }^{\text {int }} C D R 2^{\text {hi }}$ and EpCAM ${ }^{\text {hi }} \mathrm{CDR} 2^{-}$, respectively (31). AIRE and FEZF2 are also expressed in human mTECs, indicating a conserved mechanism for the regulation of tissue-restricted self-antigens $(19,32,33)$. Recent single-cell transcriptomic analyses across the lifespan showed a largely conserved TEC heterogeneity in humans (34). cTECs are more abundant during early fetal development, then a population with CTEC and mTEC properties appears in the late fetal and pediatric human thymus and lastly mTECs are dominants. Interestingly, two rare TEC subsets expressing MYOD1 and NEUROD1 genes that resemble myoid and neuroendocrine cells, respectively, were also identified. Although these subsets are preferentially located in the medulla, their respective function remains to be studied.

\section{RANK-RANKL AXIS IN MTEC EXPANSION AND DIFFERENTIATION}

The tumor necrosis factor receptor superfamily (TNFRSF) member, receptor activator of nuclear factor kappa-B (RANK; encoded by Tnfrsf11a) and its cognate ligand RANKL (encoded by Tnfsf11) play a privileged role in mTEC expansion and differentiation. During embryonic development, RANK gradually increases and is expressed by Aire $^{+}$mTEC precursors (35). In the adult, RANK is expressed by subsets that reside within mTEC $^{\text {lo }}$ and mTEC ${ }^{\text {hi }}$, including CCL2 $1^{+}$and Aire ${ }^{+}$cells (36). Importantly, the RANK-RANKL axis activates the classical and non-classical NF- $\kappa B$ signaling pathways that control the development of Aire ${ }^{+}$mTECs (37). In the embryonic thymus of RANK- or RANKL-deficient mice, Aire ${ }^{+}$mTECs are absent, indicating that this axis governs the emergence of $\mathrm{Aire}^{+}$mTECs $(37,38)$. At this stage, RANKL is provided by $\mathrm{CD}^{+} \mathrm{CD}^{-}$ lymphoid tissue inducer (LTi) cells and invariant $\mathrm{V} 5^{+}$dendritic epidermal T cells (DETC) (39). Nevertheless, other hematopoietic cells might be implicated since few Aire ${ }^{+}$mTECs are still detected in the embryonic thymus of mice lacking both LTi cells and DETC. In the postnatal thymus, the absence of RANK or RANKL leads to a partial reduction in Aire ${ }^{+}$mTECs, showing that other signal(s) are involved in mTEC differentiation after birth $(37,40)$.

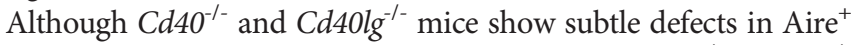
mTECs, these cells are further decreased in Tnfrsf $11 a^{-/-} \times C d 40^{-/-}$ double-deficient mice compared to Tnfrsf $11 a^{-/-}$mice, showing that RANK and CD40 cooperate to induce mTEC differentiation after birth (37). In the postnatal thymus, whereas CD40L is exclusively provided by $\mathrm{CD} 4^{+}$thymocytes, RANKL is higher in $\mathrm{CD} 4^{+}$than in $\mathrm{CD}^{+}$thymocytes and detected in iNKT cells (40-42) (Figure 1A). The contribution of LTi, DETC and iNKT cells in the adult might be limited due to their paucity compared to the large numbers of $\mathrm{CD}^{+}$thymocytes. This assumption is corroborated by the fact that mice deficient in $\mathrm{CD}_{4}^{+}$thymocytes have a dramatic reduction in Aire ${ }^{+}$mTECs and an underdeveloped medulla $(41,43)$.

RANKL is primarily synthesized as a membrane-bound trimeric complex that can be cleaved into its soluble form by proteases (44).
A recent study showed that mice lacking soluble RANKL have normal numbers of Aire ${ }^{+}$mTECs, indicating that membranebound rather than soluble RANKL induces their differentiation (45). Accordingly, RANKL and CD40L signals are delivered by $\mathrm{CD}^{+}$thymocytes in the context of antigen-specific TCR/MHCIImediated interactions with mTECs $(41,43,46)$. This is well illustrated in Rip-mOVAxOTII-Rag2 ${ }^{-/-}$mice, in which the RipmOVA transgene drives the expression of membrane-bound OVA in mTECs allowing high affinity interactions with OVA-specific OTII $\mathrm{CD}^{+}$thymocytes. Aire ${ }^{+}$mTECs develop in these mice in contrast to OTII-Rag2 ${ }^{-/-}$mice. RANKL in $\mathrm{CD}^{+}$thymocytes is likely regulated by TGF $\beta$ RII signaling (47). Mice lacking TGF $\beta$ RII in $\alpha \beta$ thymocytes at the early DP stage (Cd4-cre $\mathrm{x} T g f b r 2^{\mathrm{t} / \mathrm{fl}}$ mice) have reduced RANKL levels in Helios ${ }^{+}$autoreactive $\mathrm{CD}^{+}$thymocytes. Conversely, the stimulation of purified autoreactive $\mathrm{CD} 4^{+}$ thymocytes with TGF- $\beta$ increases RANKL expression. This upregulation is prevented by MAPK pathway inhibitors, indicating that TGF $\beta$ RII signaling induces RANKL by its SMAD4/TRIM33-independent pathway. Similarly, TGF- $\beta$ stimulation was shown to increase RANKL in TCR-activated Tcell hybridoma (48).

RANK signaling is regulated by the soluble decoy receptor for RANKL, osteoprotegerin (OPG; encoded by Tnfrsf11b), which inhibits RANKL interaction with its receptor RANK. OPG deficiency leads to an increased mTEC cellularity resulting in enlarged medulla with an enrichment in Aire ${ }^{+}$mTECs (49). Mice harboring a Tnfrsf $11 b$ deletion in mTECs have increased numbers of total and Aire ${ }^{+}$mTECs, similarly to Tnfrsf $11 b^{-/-}$mice (50). Thus, OPG produced locally by mTECs rather than serum OPG regulates mTEC cellularity and differentiation. RANK activates Aire expression by the NF- $\mathrm{\kappa B}$ signaling because Aire contains in its upstream coding region a highly conserved noncoding sequence 1 (CNS1) with two NF- $\mathrm{KB}$ binding sites $(51,52)$. CNS1-deficient mice consequently lack Aire expression in mTECs and show many characteristics of Aire $e^{-1-}$ mice including reduced Aire-dependent tissue-restricted self-antigens. Noteworthy, the RANK-RANKL axis does not only induce Aire by itself but also controls mTEC cellularity and differentiation. In addition to Aire ${ }^{+}$mTECs, Tnfsf $11^{-/}$mice show reduced numbers of mTEC $^{\text {lo }}$ and mTEC ${ }^{\text {hi }}$ (37). Conversely, Tnfrsf $11 b^{-/-}$mice have increased numbers of CCL21 $^{-}$and CCL21 ${ }^{+}$mTEC $^{\text {lo }}$ and Aire and Aire ${ }^{+}$mTEC $^{\text {hi }}$ (36, 49). Accordingly, the stimulation of 2-deoxyguanosine-treated thymic lobes with RANKL show increased mTEC cellularity including Aire ${ }^{+}$mTEC $^{\text {hi }}$, which is further augmented by the addition of CD40L protein $(43,53)$. Furthermore, in vivo antiRANKL blockade results in a severe depletion of around $80 \%$ of mTECs with a substantial loss of TTEC $^{\text {lo }}$ and Aire $^{+}$mTEC $^{\text {hi }}$ (49). In addition to control Aire ${ }^{+}$mTECs, RANK signaling therefore regulates the overall mTEC cellularity.

In humans, scRNA-seq data indicate that RANK is expressed by Aire ${ }^{+}$mTECs (34). Interestingly, the stimulation of primary human mTECs with RANKL leads to the upregulation of AIRE mRNA, suggesting a conserved role for RANK signaling (54). Given the implication of RANK-RANKL axis in bone resorption, a monoclonal antibody specific of human soluble and membranebound RANKL, Denosumab, has been developed to inhibit osteoclast development and activity. Denosumab is now used in 
A

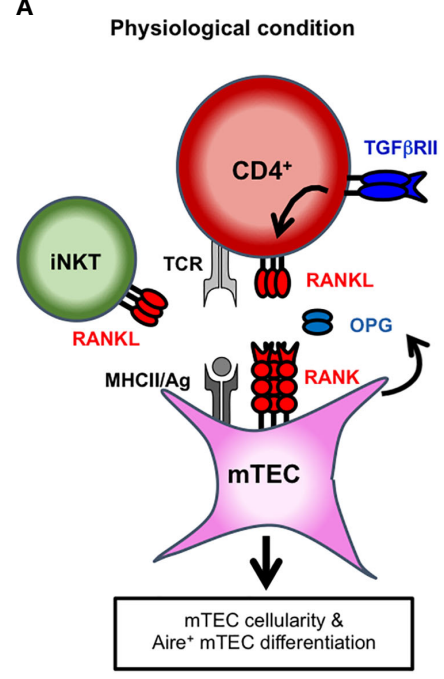

B

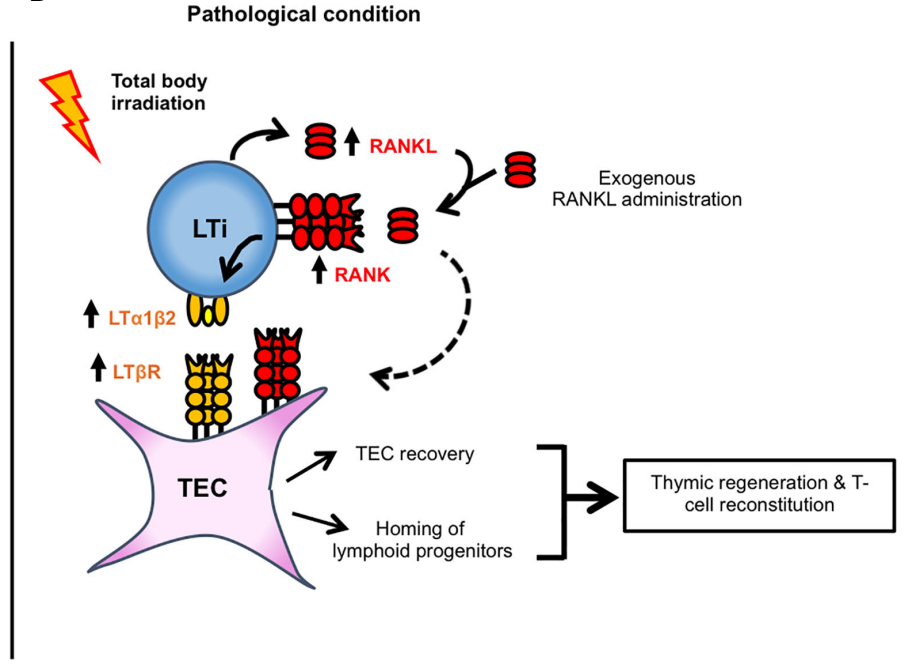

FIGURE 1 | Key cellular actors implicated in RANK-RANKL signaling axis and in mTEC development and thymic regeneration. (A) TGF $\beta R \|$ signaling upregulates the expression of RANKL in autoreactive CD4 $4^{+}$thymocytes. RANKL, expressed by autoreactive CD4 $4^{+}$thymocytes and iNKT cells, controls mTEC cellularity and Aire ${ }^{+}$ mTEC differentiation. By binding to RANKL as a decoy receptor, OPG produced by mTECs inhibits RANK signaling and thereby regulates mTEC development. (B) Upon total body irradiation, radio-resistant LTi cells and CD4 $4^{+}$thymocytes upregulate RANKL expression. LTi cells also upregulate RANK receptor. RANKL upregulation or exogenous RANKL administration induces the heterocomplex LT $\alpha 1 \beta 2$ at the cell surface of $L T i$. RANKL could also stimulate RANK signaling in TECs (dashed arrow). TECs also upregulate the corresponding LTRR receptor after total body irradiation. In turn, LT $\alpha 1 \beta 2-L T \beta R$ axis activation induces TEC regeneration by promoting their proliferation and survival. Furthermore, by inducing the expression of chemokines and adhesion molecules, this axis also favors the thymus homing of circulating T-cell progenitors.

therapy to treat osteoporosis, primary bone tumors and bone metastases $(55,56)$. Nevertheless, considering the importance of RANK-RANKL axis in Aire $^{+}$mTEC differentiation, it remains to be defined whether this treatment could affect central tolerance and increase the risk of autoimmunity.

\section{SENSIBILITY OF TECS TO MYELOABLATIVE CONDITIONING REGIMEN}

Myeloablative treatments such as radiation and chemotherapy deplete hematopoietic cells and in particular DP thymocytes that are extremely sensitive. These treatments also impair the recruitment of circulating $\mathrm{T}$-cell progenitors and induce damages on TECs (Figure 2). Consequently, the generation of newly produced naive T cells is reduced. Since TECs dictate the size of stromal niches, TEC injury contributes in a delayed T-cell reconstitution upon bone marrow (BMT) or hematopoietic stem transplantation (HSCT). In humans, allogeneic HSCT survivors are immunodeficient in $\mathrm{T}$ cells for at least 1 year, a period of high susceptibility to opportunistic infections, autoimmunity or tumor relapse, increasing the risk of morbidity and mortality $(57,58)$. Although innate cells and antibodies may limit viral infections, cytotoxic $\mathrm{CD}^{+} \mathrm{T}$ cells and helper $\mathrm{CD} 4^{+} \mathrm{T}$ cells are essential in viral clearance and the prevention of recurrent infections. T-cell recovery thus protects from lethality after BMT or HSCT. Importantly, T-cell immunity relies on the regeneration of the thymus and its capacity to produce naïve $\mathrm{T}$ cells. Total body irradiation (TBI) leads rapidly in a profound reduction of the cortex due to the loss of DP thymocytes and a substantial decrease of the medulla (59). Both cTECs and mTECs are radiosensitive $(60,61)$. Among mTECs, Aire ${ }^{+}$mature mTECs are lost upon TBI and treatment with the chemotherapy agent cyclophosphamide or the immunosuppressant cyclosporine A, used to prevent allograft rejection $(61,62)$. However, the effects of such treatments on the recently identified dozens of TEC subsets remain to be investigated. Remarkably, the injured thymic tissue retains potent regenerative capacity. Targeting the pathways implicated in endogenous TEC regeneration is expected to improve thymicdependent $\mathrm{T}$-cell recovery. Potential strategies based on keratinocyte growth factor (KGF), IL-22 or Bone Morphogenic Protein 4 (BMP4) have been reviewed in $(58,63,64)$. Strategies based on FOXN1 protein or cDNA administration also improve TEC regeneration both in the context of HSCT and aging $(65,66)$. A novel role for the RANK-RANKL axis in TEC regeneration and T-cell recovery is highlighted below.

\section{RANK-RANKL AXIS IN TEC REGENERATION}

RANKL is upregulated in radio-resistant LTi cells and CD4 ${ }^{+}$ thymocytes during the early phase of thymic regeneration after total body irradiation (TBI) $(61,67,68)$. Although LTi cells are rare in the thymus, they express a higher level of RANKL than $\mathrm{CD}^{+}$thymocytes after TBI (61). Interestingly, the administration of a neutralizing anti-RANKL antibody impairs TEC regeneration, 


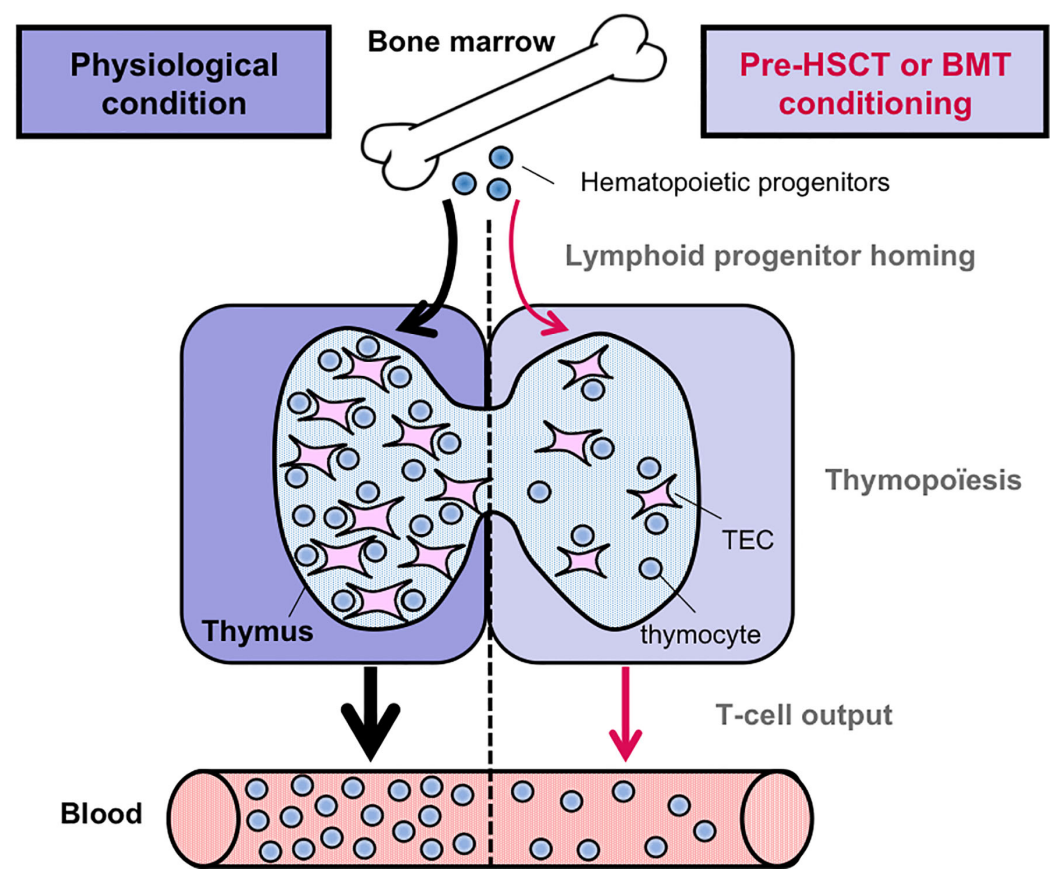

FIGURE 2 | Pre-transplantation conditioning regimen alters thymic-dependent T-cell production. In contrast to the physiological condition, the homing ability of circulating T-cell progenitors is reduced after pre-HSCT or BMT conditioning regimen. Furthermore, T-cell production is also reduced, notably due to TEC damages induced by myeloablative regimens. Consequently, the output of newly generated naïve $T$ cells is diminished.

emphasizing an important role for RANKL in endogenous TEC recovery. Conversely, RANKL protein administration increases TEC numbers at a level close to unirradiated mice. RANKL enhances cTEC and mTEC numbers, including Aire $^{+}$mTEC $^{\text {hi }}$ and TEPC-enriched cells, likely by stimulating their proliferation and survival. These observations are in agreement with a previous study indicating that RANKL increases in vitro the proliferation of cortical and medullary TEC cell lines (69). Of clinical relevance, RANKL administration upon BMT boosts not only the regeneration of several TEC subsets but also increases T-cell progenitor homing (Figure 1B) (61). This latter effect could be explained by an enhanced cellularity of endothelial cells upon RANKL administration although further investigations are required. Consequently, this treatment ameliorates de novo thymopoiesis and peripheral T-cell reconstitution. Noteworthy, a single course of RANKL after BMT boosts thymic regeneration at least during 2 months, indicative of a lasting effect. This therapeutic strategy is also efficient in aged individuals in whom T-cell recovery upon BMT is less efficient and delayed (70). Agerelated thymic involution results in a disrupted thymic architecture with a reduced TEC cellularity, which alters T-cell production (71). RANKL treatment could be thus of special interest to the elderly, although further studies are required.

Mechanistically, RANKL upregulates another TNF family ligand, lymphotoxin $\alpha$ (LT $\alpha$; encoded by Lta), expressed as a membrane anchored LT $\alpha 1 \beta 2$ heterocomplex, in LTi of recipient origin (Figure 1B) (61). Conversely, the RANK-Fc antagonist fully blocks LT $\alpha 1 \beta 2$ upregulation. Noteworthy RANKL also induces LT $\alpha 1 \beta 2$ expression in LTi cells during lymph node formation (72).
Likewise RANKL, LT $\alpha$ is upregulated during the early phase of thymic regeneration. Since $\mathrm{CD} 4^{+}$thymocytes upregulate RANKL and since LTi cells express both RANK and its ligand, RANK signaling may be triggered in LTi in an autocrine and paracrine manner. Given that LTi cells upregulate RANKL, LT $\alpha 1 \beta 2$, IL-22, IL-23R, and ROR $\gamma$ after thymic injury $(61,68)$, these cells are likely in a quiescent stage at steady state and activated after irradiation to repair the injured thymic tissue. Accordingly, the depletion of ILC3, comprising LTi cells, in an experimental model of graft-versus-host disease (GVHD) results in impaired thymic regeneration (73). Interestingly, LT $\beta \mathrm{R}$ is also upregulated in cTECs, mTECs, and TEPC-enriched cells after TBI, suggesting that the LT $\alpha 1 \beta 2-\mathrm{LT} \beta \mathrm{R}$ axis is implicated in TEC regeneration (61). At steady state, $\mathrm{Lta}^{-/-}$mice show normal numbers of TEC subsets. In contrast, cTECs, mTECs including Aire ${ }^{+}$mTEC $^{\text {hi }}$ and TEPC-enriched cells are substantially reduced in these mice upon BMT. These observations indicate that the mechanisms implicated in TEC regeneration are distinct from those used at steady state. Furthermore, these mice show reduced numbers of early T-cell progenitors (ETPs) because LT $\alpha$ controls the homing capacity of circulating $\mathrm{T}$-cell progenitors by regulating the expression of CCL19 and CCL21 in TECs and ICAM-1, VCAM-1, and Pselectin in endothelial cells, all implicated in T-cell progenitor homing $(61,74)$. Similarly, $\mathrm{Ltbr}^{-/-}$mice have an altered recruitment of T-cell progenitors after sublethal TBI (75). In agreement with defective TEC regeneration and T-cell progenitor homing, BMtransplanted $\mathrm{Lta}^{-/-}$mice have impaired thymic and peripheral Tcell reconstitution. These beneficial effects induced by RANKL depend on LT $\alpha$ since they are essentially lost when RANKL is 
administered in $\mathrm{Lta}^{-/-}$recipients. RANKL administration thus constitutes a novel therapeutic strategy to improve T-cell function recovery after thymic injury. Interestingly, RANK and $\mathrm{LT} \beta \mathrm{R}$ expression is conserved in the human thymus, opening potential therapeutic perspectives (34). Besides applications linked to myeloablative conditioning regimen, these in vivo findings open new avenues to treat patients whose thymus has been severely damaged by aging, viral infections, or malnutrition.

\section{AUTHOR CONTRIBUTIONS}

The author confirms being the sole contributor of this work and has approved it for publication.

\section{REFERENCES}

1. Irla M. Thymic crosstalk: an overview of the complex cellular interactions that controls the establishment of T-cell tolerance. In Passos, GA. Editor. Chapter 6 Thymus Transcriptome and Cell Biology. Cham: Springer Nature (2019). pp. 318. doi: 10.1007/978-3-030-12040-5_6

2. Lopes N, Serge A, Ferrier P, Irla M. Thymic Crosstalk Coordinates Medulla Organization and T-Cell Tolerance Induction. Front Immunol (2015) 6:365. doi: 10.3389/fimmu.2015.00365

3. van Ewijk W, Shores EW, Singer A. Crosstalk in the mouse thymus. Immunol Today (1994) 15(5):214-7. doi: 10.1016/0167-5699(94)90246-1

4. Klug DB, Carter C, Crouch E, Roop D, Conti CJ, Richie ER. Interdependence of cortical thymic epithelial cell differentiation and T-lineage commitment. Proc Natl Acad Sci U S A (1998) 95(20):11822-7. doi: 10.1073/pnas.95.20. 11822

5. Gossens K, Naus S, Corbel SY, Lin S, Rossi FM, Kast J, et al. Thymic progenitor homing and lymphocyte homeostasis are linked via S1Pcontrolled expression of thymic P-selectin/CCL25. J Exp Med (2009) 206 (4):761-78. doi: 10.1084/jem.20082502

6. Plotkin J, Prockop SE, Lepique A, Petrie HT. Critical role for CXCR4 signaling in progenitor localization and $\mathrm{T}$ cell differentiation in the postnatal thymus. J Immunol (2003) 171(9):4521-7. doi: 10.4049/ jimmunol.171.9.4521

7. Hozumi K, Mailhos C, Negishi N, Hirano K, Yahata T, Ando K, et al. Deltalike 4 is indispensable in thymic environment specific for $\mathrm{T}$ cell development. J Exp Med (2008) 205(11):2507-13. doi: 10.1084/jem.20080134

8. Koch U, Fiorini E, Benedito R, Besseyrias V, Schuster-Gossler K, Pierres M, et al. Delta-like 4 is the essential, nonredundant ligand for Notch1 during thymic T cell lineage commitment. J Exp Med (2008) 205(11):2515-23. doi: 10.1084 /jem.20080829

9. Alves NL, Richard-Le Goff O, Huntington ND, Sousa AP, Ribeiro VS, Bordack A, et al. Characterization of the thymic IL-7 niche in vivo. Proc Natl Acad Sci U S A (2009) 106(5):1512-7. doi: 10.1073/pnas.0809559106

10. Ohigashi I, Kozai M, Takahama Y. Development and developmental potential of cortical thymic epithelial cells. Immunol Rev (2016) 271(1):10-22. doi: 10.1111/imr.12404

11. Xing Y, Jameson SC, Hogquist KA. Thymoproteasome subunit- $E \leq 5 \mathrm{~T}$ generates peptide-MHC complexes specialized for positive selection. Proc Natl Acad Sci U S A (2013) 110(17):6979-84. doi: 10.1073/pnas.1222244110

12. Baran-Gale J, Morgan MD, Maio S, Dhalla F, Calvo-Asensio I, Deadman ME, et al. Ageing compromises mouse thymus function and remodels epithelial cell differentiation. Elife (2020) 9. doi: 10.7554/eLife.56221

13. Wekerle H, Ketelsen UP, Ernst M. Thymic nurse cells. Lymphoepithelial cell complexes in murine thymuses: morphological and serological characterization. J Exp Med (1980) 151(4):925-44. doi: 10.1084/jem.151.4.925

14. Nakagawa Y, Ohigashi I, Nitta T, Sakata M, Tanaka K, Murata S, et al. Thymic nurse cells provide microenvironment for secondary $\mathrm{T}$ cell receptor alpha rearrangement in cortical thymocytes. Proc Natl Acad Sci U S A (2012) 109 (50):20572-7. doi: 10.1073/pnas.1213069109

\section{FUNDING}

This work received funding from the Agence Nationale de la Recherche (grant ANR-19-CE18-0021-01, RANKLthym to MI) and was supported by institutional grants from Institut National de la Santé et de la Recherche Médicale, Centre National de la Recherche Scientifique and Aix-Marseille Université.

\section{ACKNOWLEDGMENTS}

I gratefully thank Pr Arnauld Sergé (LAI, Marseille) for critical reading of the manuscript.

15. Hollander GA, Wang B, Nichogiannopoulou A, Platenburg PP, van Ewijk W, Burakoff SJ, et al. Developmental control point in induction of thymic cortex regulated by a subpopulation of prothymocytes. Nature (1995) 373 (6512):350-3. doi: 10.1038/373350a0

16. Shakib S, Desanti GE, Jenkinson WE, Parnell SM, Jenkinson EJ, Anderson G. Checkpoints in the development of thymic cortical epithelial cells. J Immunol (2009) 182(1):130-7. doi: 10.4049/jimmunol.182.1.130

17. van Ewijk W, Hollander G, Terhorst C, Wang B. Stepwise development of thymic microenvironments in vivo is regulated by thymocyte subsets. Development (2000) 127(8):1583-91.

18. Sansom SN, Shikama-Dorn N, Zhanybekova S, Nusspaumer G, Macaulay IC, Deadman ME, et al. Population and single-cell genomics reveal the Aire dependency, relief from Polycomb silencing, and distribution of self-antigen expression in thymic epithelia. Genome Res (2014) 24(12):1918-31. doi: $10.1101 /$ gr.171645.113

19. Takaba H, Morishita Y, Tomofuji Y, Danks L, Nitta T, Komatsu N, et al. Fezf2 Orchestrates a Thymic Program of Self-Antigen Expression for Immune Tolerance. Cell (2015) 163(4):975-87. doi: 10.1016/j.cell.2015.10.013

20. Gray DH, Seach N, Ueno T, Milton MK, Liston A, Lew AM, et al. Developmental kinetics, turnover, and stimulatory capacity of thymic epithelial cells. Blood (2006) 108(12):3777-85. doi: 10.1182/blood-2006-02004531

21. Gabler J, Arnold J, Kyewski B. Promiscuous gene expression and the developmental dynamics of medullary thymic epithelial cells. Eur J Immunol (2007) 37(12):3363-72. doi: 10.1002/eji.200737131

22. Gray D, Abramson J, Benoist C, Mathis D. Proliferative arrest and rapid turnover of thymic epithelial cells expressing Aire. J Exp Med (2007) 204 (11):2521-8. doi: 10.1084/jem.20070795

23. Wong K, Lister NL, Barsanti M, Lim JM, Hammett MV, Khong DM, et al. Multilineage potential and self-renewal define an epithelial progenitor cell population in the adult thymus. Cell Rep (2014) 8(4):1198-209. doi: 10.1016/ j.celrep.2014.07.029

24. Kozai M, Kubo Y, Katakai T, Kondo H, Kiyonari H, Schaeuble K, et al. Essential role of CCL21 in establishment of central self-tolerance in T cells. J Exp Med (2017) 214(7):1925-35. doi: 10.1084/jem.20161864

25. Nishikawa Y, Hirota F, Yano M, Kitajima H, Miyazaki J, Kawamoto H, et al. Biphasic Aire expression in early embryos and in medullary thymic epithelial cells before end-stage terminal differentiation. J Exp Med (2010) 207(5):96371. doi: 10.1084/jem.20092144

26. Metzger TC, Khan IS, Gardner JM, Mouchess ML, Johannes KP, Krawisz AK, et al. Lineage tracing and cell ablation identify a post-Aire-expressing thymic epithelial cell population. Cell Rep (2013) 5(1):166-79. doi: 10.1016/ j.celrep.2013.08.038

27. Michel C, Miller CN, Kuchler R, Brors B, Anderson MS, Kyewski B, et al. Revisiting the Road Map of Medullary Thymic Epithelial Cell Differentiation. J Immunol (2017) 199(10):3488-503. doi: 10.4049/jimmunol.1700203

28. Bornstein C, Nevo S, Giladi A, Kadouri N, Pouzolles M, Gerbe F, et al. Singlecell mapping of the thymic stroma identifies IL-25-producing tuft epithelial cells. Nature (2018) 559(7715):622-6. doi: 10.1038/s41586-018-0346-1 
29. Miller CN, Proekt I, von Moltke J, Wells KL, Rajpurkar AR, Wang H, et al. Thymic tuft cells promote an IL-4-enriched medulla and shape thymocyte development. Nature (2018) 559(7715):627-31. doi: 10.1038/s41586-0180345-2

30. Dhalla F, Baran-Gale J, Maio S, Chappell L, Hollander GA, Ponting CP. Biologically indeterminate yet ordered promiscuous gene expression in single medullary thymic epithelial cells. EMBO J (2020) 39(1):e101828. doi: 10.15252/embj.2019101828

31. Gotter J, Brors B, Hergenhahn M, Kyewski B. Medullary epithelial cells of the human thymus express a highly diverse selection of tissue-specific genes colocalized in chromosomal clusters. J Exp Med (2004) 199(2):155-66. doi: 10.1084/jem.20031677

32. Dragin N, Bismuth J, Cizeron-Clairac G, Biferi MG, Berthault C, Serraf A, et al. Estrogen-mediated downregulation of AIRE influences sexual dimorphism in autoimmune diseases. J Clin Invest (2016) 126(4):1525-37. doi: 10.1172/JCI81894

33. Pinto S, Michel C, Schmidt-Glenewinkel H, Harder N, Rohr K, Wild S, et al. Overlapping gene coexpression patterns in human medullary thymic epithelial cells generate self-antigen diversity. Proc Natl Acad Sci U S A (2013) 110(37):E3497-505. doi: 10.1073/pnas.1308311110

34. Park JE, Botting RA, Dominguez Conde C, Popescu DM, Lavaert M, Kunz DJ, et al. A cell atlas of human thymic development defines $\mathrm{T}$ cell repertoire formation. Science (2020) 367(6480):eaay3224. doi: 10.1126/science.aay3224

35. Akiyama N, Takizawa N, Miyauchi M, Yanai H, Tateishi R, Shinzawa M, et al. Identification of embryonic precursor cells that differentiate into thymic epithelial cells expressing autoimmune regulator. J Exp Med (2016) 213 (8):1441-58. doi: 10.1084/jem.20151780

36. McCarthy NI, Cowan JE, Nakamura K, Bacon A, Baik S, White AJ, et al. Osteoprotegerin-Mediated Homeostasis of Rank+ Thymic Epithelial Cells Does Not Limit Foxp3+ Regulatory T Cell Development. J Immunol (2015) 195(6):2675-82. doi: 10.4049/jimmunol.1501226

37. Akiyama T, Shimo Y, Yanai H, Qin J, Ohshima D, Maruyama Y, et al. The tumor necrosis factor family receptors RANK and CD40 cooperatively establish the thymic medullary microenvironment and self-tolerance. Immunity (2008) 29(3):423-37. doi: 10.1016/j.immuni.2008.06.015

38. Rossi SW, Kim MY, Leibbrandt A, Parnell SM, Jenkinson WE, Glanville SH, et al. RANK signals from $\mathrm{CD} 4(+) 3(-)$ inducer cells regulate development of Aire-expressing epithelial cells in the thymic medulla. J Exp Med (2007) 204 (6):1267-72. doi: 10.1084/jem.20062497

39. Roberts NA, White AJ, Jenkinson WE, Turchinovich G, Nakamura K, Withers $\mathrm{DR}$, et al. Rank signaling links the development of invariant gammadelta $\mathrm{T}$ cell progenitors and Aire(+) medullary epithelium. Immunity (2012) 36(3):42737. doi: 10.1016/j.immuni.2012.01.016

40. Hikosaka Y, Nitta T, Ohigashi I, Yano K, Ishimaru N, Hayashi Y, et al. The cytokine RANKL produced by positively selected thymocytes fosters medullary thymic epithelial cells that express autoimmune regulator. Immunity (2008) 29(3):438-50. doi: 10.1016/j.immuni.2008.06.018

41. Irla M, Hugues S, Gill J, Nitta T, Hikosaka Y, Williams IR, et al. Autoantigenspecific interactions with CD4+ thymocytes control mature medullary thymic epithelial cell cellularity. Immunity (2008) 29(3):451-63. doi: 10.1016/ j.immuni.2008.08.007

42. White AJ, Jenkinson WE, Cowan JE, Parnell SM, Bacon A, Jones ND, et al. An essential role for medullary thymic epithelial cells during the intrathymic development of invariant NKT cells. J Immunol (2014) 192(6):2659-66. doi: 10.4049 /jimmunol.1303057

43. Irla M, Guerri L, Guenot J, Serge A, Lantz O, Liston A, et al. Antigen recognition by autoreactive $\mathrm{cd} 4(+)$ thymocytes drives homeostasis of the thymic medulla. PloS One (2012) 7(12):e52591. doi: 10.1371/journal.pone. 0052591

44. Lum L, Wong BR, Josien R, Becherer JD, Erdjument-Bromage H, Schl $\sqrt{ } \partial$ ndorff $J$, et al. Evidence for a role of a tumor necrosis factor-alpha (TNF-alpha)-converting enzyme-like protease in shedding of TRANCE, a TNF family member involved in osteoclastogenesis and dendritic cell survival. J Biol Chem (1999) 274(19):13613-8. doi: 10.1074/jbc.274.19.13613

45. Asano T, Okamoto K, Nakai Y, Tsutsumi M, Muro R, Suematsu A, et al. Soluble RANKL is physiologically dispensable but accelerates tumour metastasis to bone. Nat Metab (2019) 1(9):868-75. doi: 10.1038/s42255019-0104-1
46. Irla M, Hollander G, Reith W. Control of central self-tolerance induction by autoreactive CD4+ thymocytes. Trends Immunol (2010) 31(2):71-9. doi: 10.1016/j.it.2009.11.002

47. McCarron MJ, Irla M, Serge A, Soudja SM, Marie JC. Transforming Growth Factor-beta signaling in alphabeta thymocytes promotes negative selection. Nat Commun (2019) 10(1):5690. doi: 10.1038/s41467-019-13456-z

48. Wang R, Zhang L, Zhang X, Moreno J, Celluzzi C, Tondravi M, et al. Regulation of activation-induced receptor activator of NF-kappaB ligand (RANKL) expression in T cells. Eur J Immunol (2002) 32(4):1090-8. doi: 10.1002/1521-4141(200204)32:4<1090::aid-immu1090>3.0.co;2-p

49. Khan IS, Mouchess ML, Zhu ML, Conley B, Fasano KJ, Hou Y, et al. Enhancement of an anti-tumor immune response by transient blockade of central T cell tolerance. J Exp Med (2014) 211(5):761-8. doi: 10.1084/ jem.20131889

50. Tsukasaki M, Asano T, Muro R, Huynh NC, Komatsu N, Okamoto K, et al. OPG Production Matters Where It Happened. Cell Rep (2020) 32(10):108124. doi: 10.1016/j.celrep.2020.108124

51. Haljasorg U, Bichele R, Saare M, Guha M, Maslovskaja J, K $\sqrt{\mu n d} \mathrm{~K}$, et al. A highly conserved NF- $E \int B$-responsive enhancer is critical for thymic expression of Aire in mice. Eur J Immunol (2015) 45(12):3246-56. doi: 10.1002/eji.201545928

52. LaFlam TN, Seumois G, Miller CN, Lwin W, Fasano KJ, Waterfield M, et al. Identification of a novel cis-regulatory element essential for immune tolerance. J Exp Med (2015) 212(12):1993-2002. doi: 10.1084/jem.20151069

53. Mouri Y, Yano M, Shinzawa M, Shimo Y, Hirota F, Nishikawa $Y$, et al. Lymphotoxin Signal Promotes Thymic Organogenesis by Eliciting RANK Expression in the Embryonic Thymic Stroma. J Immunol (2011) 193:4356-67. doi: 10.4049 /jimmunol.1003533

54. Villegas JA, Gradolatto A, Truffault F, Roussin R, Berrih-Aknin S, Le Panse R, et al. Cultured Human Thymic-Derived Cells Display Medullary Thymic Epithelial Cell Phenotype and Functionality. Front Immunol (2018) 9:1663. doi: 10.3389/fimmu.2018.01663

55. Sobacchi C, Menale C, Villa A. The RANKL-RANK Axis: A Bone to Thymus Round Trip. Front Immunol (2019) 10:629. doi: 10.3389/fimmu. 2019.00629

56. Ahern E, Smyth MJ, Dougall WC, Teng MWL. Roles of the RANKL-RANK axis in antitumour immunity - implications for therapy. Nat Rev Clin Oncol (2018) 15(11):676-93. doi: 10.1038/s41571-018-0095-y

57. Mackall C, Fry T, Gress R, Peggs K, Storek J, Toubert A. Background to hematopoietic cell transplantation, including post transplant immune recovery. Bone Marrow Transplant (2009) 44(8):457-62. doi: 10.1038/ bmt.2009.255

58. Chaudhry MS, Velardi E, Malard F, van den Brink MR. Immune Reconstitution after Allogeneic Hematopoietic Stem Cell Transplantation: Time To T Up the Thymus. J Immunol (2017) 198(1):40-6. doi: 10.4049/ jimmunol.1601100

59. Irla M, Guenot J, Sealy G, Reith W, Imhof BA, Serge A. Three-dimensional visualization of the mouse thymus organization in health and immunodeficiency. J Immunol (2013) 190(2):586-96. doi: 10.4049/ jimmunol.1200119

60. Kelly RM, Goren EM, Taylor PA, Mueller SN, Stefanski HE, Osborn MJ, et al. Short-term inhibition of p53 combined with keratinocyte growth factor improves thymic epithelial cell recovery and enhances T-cell reconstitution after murine bone marrow transplantation. Blood (2010) 115(5):1088-97. doi: 10.1182/blood-2009-05-223198

61. Lopes N, Vachon H, Marie J, Irla M. Administration of RANKL boosts thymic regeneration upon bone marrow transplantation. EMBO Mol Med (2017) 9 (6):835-51. doi: 10.15252/emmm.201607176

62. Fletcher AL, Lowen TE, Sakkal S, Reiseger JJ, Hammett MV, Seach N, et al. Ablation and regeneration of tolerance-inducing medullary thymic epithelial cells after cyclosporine, cyclophosphamide, and dexamethasone treatment. J Immunol (2009) 183(2):823-31. doi: 10.4049/jimmunol.0900225

63. Kinsella S, Dudakov JA. When the Damage Is Done: Injury and Repair in Thymus Function. Front Immunol (2020) 11:1745. doi: 10.3389/fimmu.2020. 01745

64. Hollander GA, Krenger W, Blazar BR. Emerging strategies to boost thymic function. Curr Opin Pharmacol (2010) 10(4):443-53. doi: 10.1016/j.coph. 2010.04.008 
65. Song Y, Su M, Zhu J, Di W, Liu Y, Hu R, et al. FOXN1 recombinant protein enhances T-cell regeneration after hematopoietic stem cell transplantation in mice. Eur J Immunol (2016) 46(6):1518-28. doi: 10.1002/eji.201546196

66. Sun L, Guo J, Brown R, Amagai T, Zhao Y, Su DM. Declining expression of a single epithelial cell-autonomous gene accelerates age-related thymic involution. Aging Cell (2010) 9(3):347-57. doi: 10.1111/j.1474-9726.2010. 00559.x

67. Lee HW, Kim BS, Kim HJ, Lee CW, Yoo HJ, Kim JB, et al. Upregulation of receptor activator of nuclear factor-kappaB ligand expression in the thymic subcapsular, paraseptal, perivascular, and medullary epithelial cells during thymus regeneration. Histochem Cell Biol (2005) 123(4-5):491-500. doi: 10.1007/s00418-005-0751-z

68. Dudakov JA, Hanash AM, Jenq RR, Young LF, Ghosh A, Singer NV, et al. Interleukin-22 drives endogenous thymic regeneration in mice. Science (2012) 336(6077):91-5. doi: 10.1126/science.1218004

69. Lee HW, Park HK, Na YJ, Kim CD, Lee JH, Kim BS, et al. RANKL stimulates proliferation, adhesion and IL-7 expression of thymic epithelial cells. Exp Mol Med (2008) 40(1):59-70. doi: 10.3858/emm.2008.40.1.59

70. Toubert A, Glauzy S, Douay C, Clave E. Thymus and immune reconstitution after allogeneic hematopoietic stem cell transplantation in humans: never say never again. Tissue Antigens (2012) 79(2):83-9. doi: 10.1111/j.1399-0039.2011.01820.x

71. Palmer DB. The effect of age on thymic function. Front Immunol (2013) 4:316. doi: 10.3389/fimmu.2013.00316

72. Yoshida H, Naito A, Inoue J, Satoh M, Santee-Cooper SM, Ware CF, et al. Different cytokines induce surface lymphotoxin-alphabeta on IL-7 receptor- alpha cells that differentially engender lymph nodes and Peyer's patches. Immunity (2002) 17(6):823-33. doi: 10.1016/S1074-7613(02)00479-X

73. Dudakov JA, Mertelsmann AM, O'Connor MH, Jenq RR, Velardi E, Young LF, et al. Loss of thymic innate lymphoid cells leads to impaired thymopoiesis in experimental graft-versus-host disease. Blood (2017) 130 (7):933-42. doi: 10.1182/blood-2017-01-762658

74. Scimone ML, Aifantis I, Apostolou I, von Boehmer H, von Andrian UH. A multistep adhesion cascade for lymphoid progenitor cell homing to the thymus. Proc Natl Acad Sci U S A (2006) 103(18):7006-11. doi: 10.1073/ pnas.0602024103

75. Shi Y, Wu W, Chai Q, Li Q, Hou Y, Xia H, et al. LTbetaR controls thymic portal endothelial cells for haematopoietic progenitor cell homing and T-cell regeneration. Nat Commun (2016) 7:12369. doi: 10.1038/ ncomms 12369

Conflict of Interest: The author declares that the research was conducted in the absence of any commercial or financial relationships that could be construed as a potential conflict of interest.

Copyright (c) 2021 Irla. This is an open-access article distributed under the terms of the Creative Commons Attribution License (CC BY). The use, distribution or reproduction in other forums is permitted, provided the original author(s) and the copyright owner(s) are credited and that the original publication in this journal is cited, in accordance with accepted academic practice. No use, distribution or reproduction is permitted which does not comply with these terms. 